\title{
Incidence and Survival of Pediatric Soft Tissue Sarcomas in Moscow Region, Russian Federation, 2000-2009
}

\author{
D. Y. Kachanov, ${ }^{1,2}$ K. V. Dobrenkov, ${ }^{2}$ R. T. Abdullaev, ${ }^{2}$ \\ T. V. Shamanskaya, ${ }^{2}$ and S. R. Varfolomeeva ${ }^{1,2}$ \\ ${ }^{1}$ Department of Oncology and Hematology, Russian State Medical University, 1 Ostrovityanova Street, Moscow 117997, Russia \\ ${ }^{2}$ Department of Clinical Oncology, Federal Research Center of Pediatric Hematology, Oncology and Immunology, \\ 1 Samory Mashela Street, Moscow 117198, Russia
}

Correspondence should be addressed to K. V. Dobrenkov, dobrenkov@hotmail.com

Received 30 May 2011; Revised 9 January 2012; Accepted 15 January 2012

Academic Editor: Ajay Puri

Copyright () 2012 D. Y. Kachanov et al. This is an open access article distributed under the Creative Commons Attribution License, which permits unrestricted use, distribution, and reproduction in any medium, provided the original work is properly cited.

\begin{abstract}
The aim of the study was to assess the incidence and survival rates of soft tissue sarcomas (STSs) in children 0-14 years of age in Moscow Region, Russian Federation. The database of childhood population-based cancer registry of Moscow Region was used as a data source. Tumors were stratified according to International Classification of Childhood Cancer, 3d ed. Sixty-eight cases of STS were registered from 2000 to 2009. Crude incidence rate was 0,78, and age-standardized incidence rate using World Standard Population was 0,81 per 100.000 children/year. The highest age-specific incidence was observed in infants: 1,76 per 100.000 children/year. Rhabdomyosarcoma (RMS) was the most common histological type comprising 54,4\% of all STS. 5-year observed survival (OS) of all patients with STS was 64,1 (95\% CI 55,0-73,2). There was no statistically significant difference in OS between RMS - 59,2 (95\% CI 47,0-71,4) and nonrhabdomyosarcoma STS-69,3 (95\% CI 55,8-82,8) $(P=0.63)$. Incidence and survival rates of STS observed in the study were comparable to the other Eastern European countries.
\end{abstract}

\section{Introduction}

Soft tissue sarcomas (STSs) represent a heterogeneous group of neoplasms originating from primitive mesenchymal cells [1]. Population-based data suggest that these tumors comprise $4-8 \%$ of all cancer cases in patients under the age of 15 years [2-4]. STSs represent the second most common extracranial solid tumor in children in Europe and the USA following neuroblastoma $[3,4]$. STSs are divided into two distinct groups: rhabdomyosarcoma (RMS) and nonrhabdomyosarcoma STS (NRSTS) [1]. RMS is the single most frequent childhood STS, accounting for more than 50\% of cases. NRSTS represents biologically and clinically heterogeneous assortment tumors. Distribution of subgroups of STS is age dependent. RMS is more common in children less than 10 years of age whereas NRSTS predominates in older age groups [1].

Large international epidemiological studies have shown that the incidence of STS varies in different geographic regions and ethnic groups [2]. STS epidemiology in children in Russian Federation (RF) has not been adequately studied due to several reasons. The nationwide system of cancer registration established in RF in 1953 stratifies malignancies by topography of primary tumor. International classifications specifically designed for children (e.g., International Classification of Childhood Cancer, 3d eds.-ICCC-3) and based on the histogenesis of tumors are not currently used for data analysis and reporting. At the same time STS in childhood can occur in a wide range of primary sites and are of many histological types. Moreover, there is a lack of information on the survival rates of children with cancer in RF. In order to overcome these limitations a childhood population-based cancer registry was established in Moscow Region (MR) in 2000.

The aim of this study is to describe incidence and survival rates of STS in children 0-14 years old in MR for the period from 2000 to 2009. The data were analyzed for the whole group of STS and separately for RMS and NRSTS subgroups. 


\section{Materials and Methods}

Data on patients were retrieved from the database of childhood population-based cancer registry of $\mathrm{MR}$ that has prospectively registered all cancer cases in children 0-14 years old since 2000. MR is located in the central part of RF and occupies $46.000 \mathrm{~km}^{2}$ with total population of 6,6 million (2008). The region is divided in 72 municipal districts (okrug) which are clustered in 12 medical districts. Moscow city is not an administrative part of the region, and its territory is not covered by the cancer registry of MR.

All patients 0-14 years old with STS diagnosed in 20002009 were included in analysis. Case finding of patients diagnosed before 2000 allowed to exclude prevalent cases and include in the final analysis patients with STS diagnosed in 2000. The lag time from diagnosis to recording in the registry was estimated to be less than 1 year.

Multiple sources of information on new cancer cases were used and included but not limited to discharge letters from cancer hospitals, data from pathology laboratories, and from general cancer registry of Moscow Region. By the national law oncologists are obliged to fill a notification form on every newly diagnosed case of cancer. The cancer registry has an access to the database of Ministry of Healthcare of Moscow Region that contains information on patients referred to federal cancer centers. The cancer registry closely collaborates with primary care pediatricians. Notification forms for newly diagnosed patients and for patients who finished anticancer therapy were specially designed for data retrieval. These forms represent a legal document of the mandatory annual report from pediatricians of municipal districts. Followup information was collected by active search in hospital databases and by passive retrieval of notification form filled by primary care pediatricians. Identifiable death certificates were not available to the registry. Depersonalized cancer mortality database of MR was used, and special requests are sent to primary care pediatricians to trace back other records for these patients. The completeness of registration exceeded $90 \%$.

For each registered case, information included civil status profile (name, surname, gender, date of birth, and address at the time of cancer diagnosis) and disease profile (incidence date, primary site and histology, diagnostic basis, stage, and type of therapy). Diagnoses were confirmed by reference pathology laboratory in all cases. None of the cases were registered from death certificate only. Multiple primary tumors in the same patient were separately registered. The localization of the primary tumor and histological type was defined in accordance to International Classification of Diseases for Oncology (ICD-O), 3rd edition [5]. Subsequently, tumors were grouped according to International Classification of Childhood Cancer, 3rd ed. (ICCC-3) [6]. ICCC-3 refers STS to IX diagnostic group divided into 5 subgroups: IXa: RMS, IXb: fibrosarcomas, peripheral nerve sheath tumors, and other fibrous neoplasms (further in textsubgroup of "fibrosarcoma"), IXc: Kaposi sarcoma, IXd: other specified STS, and IXe: unspecified STS. For further analysis, STSs were divided into RMS (IXa subgroup) and NRSTS (IXb, IXc, IXd, and IXe subgroups) [6].
TABLE 1: Definition of the topographic categories of STS.

\begin{tabular}{ll}
\hline Site of primary tumor & ICD-O-3 topography code [5] \\
\hline \multirow{3}{*}{ Head and neck } & C00.0-C14.8, C30.0-C32.9, \\
& C41.0-C41.1, C44.0, C44.2-C44.4, \\
Orbit & C47.0, C49.0, C76.0 \\
Pelvis & C69.0-C69.9, C44.1 \\
& C41.4, C47.5, C49.5, C76.3 \\
Genitourinary system & C51.0-C57.9, C60.0-C60.9, C61.9, \\
& C67.0-C63.9, C64.9-C66.9, \\
Thorax & C49.3, C76.1 \\
Limbs & C40.0-C40.9, C44.6, C44.7, C47.1, \\
Not otherwise specified & C47.2, C49.1, C49.2, C76.4, C76.5 \\
Cthers & All codes not listed in another \\
& category \\
\hline
\end{tabular}

For classification of the primary tumor localization, ICDO-3 topography code was grouped in 8 sites [7] (Table 1).

Most of the registered patients $(45 / 68,66,2 \%)$ were treated according to consecutive protocols of the Cooperative Soft Tissue Sarcoma Study Group (Cooperative Weichteilsarkom-Studie, CWS) of the German Society of Pediatric Oncology and Haematology (GPOH) [8]. The rest of the patients received anticancer treatment according to institutional protocols that included multiagent chemotherapy, radiation therapy, and surgery.

Annual population estimates in MR were based on the data of RF population census in 2002 and were received from Moscow Regional Committee of Federal State Statistics Service. The average annual population in the given period was 876.695 (95\% CI: 843.717 to 909.673) for children aged $0-14$ years. A decrease in childhood population from 976.795 in 2000 to 868.932 in 2009 was observed during the study period. Crude annual incidence rate was calculated per 100.000 children/year. Age-standardized incidence rate (ASR) was calculated by direct method per 100.000 children/year using the World Standard Population [9]. Crude incidence rate and ASR were calculated for children 014 years old. Age-specific incidence rate was calculated for four different age groups: $<1,1-4,5-9$, and $10-14$ years. Kaplan-Meier method was used for survival analyses [10]. Observed survival was calculated for the entire group of patients, for the age groups $0-4,5-9,10-14$ years, and separately for patients with RMS and NRSTS. Differences in survival rates between various patient groups were evaluated by comparison of entire survival curves and tested with the log-rank tests. Fisher's exact test was used to compare categorical variables due to small number of cases. Statistica 8.0 (Statsoft, USA) was used for statistical analysis of the data.

\section{Results}

Sixty-eight cases of STS in children 0-14 years old were registered in 2000-2009, comprising 5,8\% of all malignant tumors in this age group. STS was the third most frequent 
extracranial solid tumor in children after neuroblastoma and renal tumors. There was a slight male prevalence with the male-to-female ratio which was $1,52: 1$. Most cases were observed in 10-14 age group (22 patients, 32,4\%). 20 $(29,4 \%)$ and $16(23,5 \%)$ cases were registered in $1-4$ and $5-$ 9 age groups, respectively. The smallest number of patients was registered in infants-10 (14,7\%) cases. The median age at diagnosis was 5 years.

Distribution of STS by diagnostic subgroup according to ICCC-3 showed the prevalence of subgroup "RMS" (Table 2), followed by subgroups of "other specified STS" and "fibrosarcoma". Kaposi sarcoma cases were not identified.

RMS was the most common histological type of STS, representing $54,4 \%$ of cases. Among NRSTS there were 8 $(11,8 \%)$ cases of sinovial sarcoma, $7(10,3 \%)$ malignant peripheral nerve sheath tumor, and $5(7,4 \%)$ cases of Ewing sarcoma family. Analysis of distribution of STS cases according to age demonstrated a negative correlation between number of RMS and patient's age: RMS comprised $70 \%$ of STS cases in infants and $80 \%$ in children $1-4$ years of age, but only $23 \%$ in $10-14$-year age group.

Location of primary tumor differed between RMS and NRSTS. Majority of RMS (43.3\%) was located in the head and neck region (including orbit) whereas NRSTS mainly affected limbs (45.2\%) (Table 3).

The crude incidence rate of STS in children 0-14 years old was 0,78 ASR was 0,81 per 100.000 children/year (Table 2). The highest age-specific incidence rate was observed in infants-1,76 per 100.000 children/year. Incidence rate of STS subgroups varied in different age groups. Age-specific incidence rate of RMS (IXa) was the highest in infants, decreasing in older age groups. Incidence rate of "other specified STS" (IXd) showed bimodal distribution with the highest incidence observed in infants and children $10-14$ years old.

Cancer predisposition syndromes presented by neurofibromatosis type 1 were observed in $3(4,4 \%)$ cases. In 1 case RMS developed as a second malignant neoplasm in the radiation field 3,4 years after the diagnosis of hereditary retinoblastoma.

The OS of all patients with STS at 5 years was 64,1 (95\% CI 55,0-73,2) (Figure 1).

There was no statistically significant difference in 5-year OS between RMS - 59,2 (95\% CI 47,0-71,4) and NRSTS69,3 (95\% CI 55,8-82,8) $(P=0,63)$ (Figure 2).

The better 5-year OS rate was observed in patient 5-9 years of age $80,0 \%$ ( $95 \%$ CI $62,5-97,5)$. The 5 -year OS for patients $0-4$ and $10-14$ years of age was $58,3 \%$ (95\% CI $44,8-$ 71,8 ), and $62,3 \%$ (95\% CI 46,3-78,3), respectively. Five-year OS in age groups $<1,1-9$ and $>10$ years was $50,0 \%(95 \%$ CI 28,1-71,9), 70,0\% (95\% CI 57,5-82,5) and 62,2\% (95\% CI $46,3-78,1)$, respectively. Difference in survival between age groups was not statistically significant.

\section{Discussion}

The present study describes the incidence and survival of STS in children in MR, the second most populated region of RF.

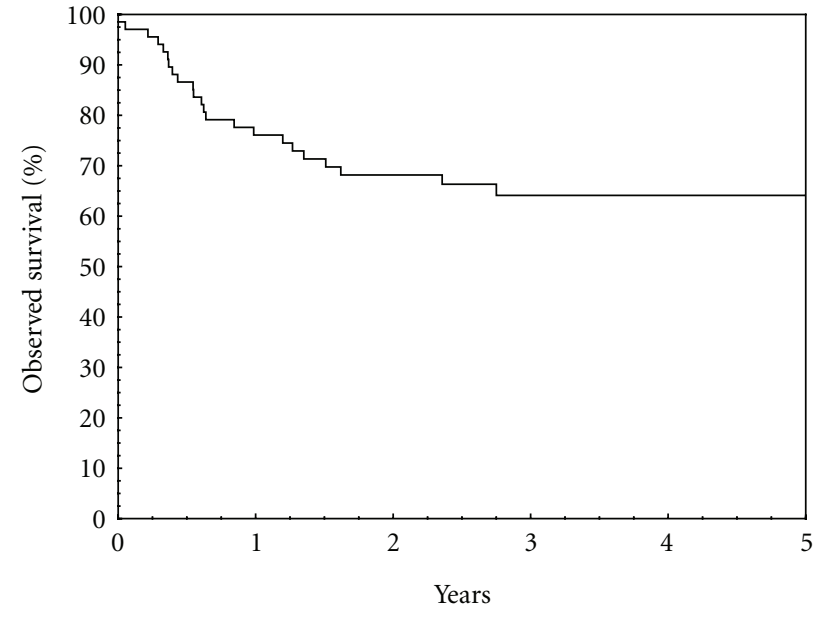

FIGURE 1: Observed survival, all patients. OS $=64,1(95 \%$ CI 55,0$73,2)$ at 5 years.

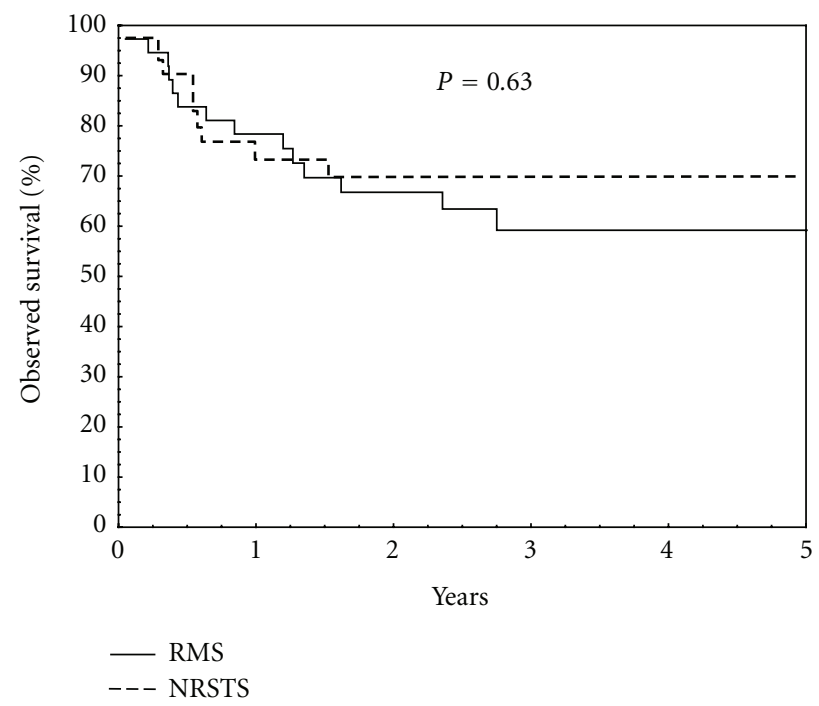

FIGURE 2: Observed survival, RMS versus NRSTS. OS for RMS = $59,2(95 \%$ CI $47,0-71,4)$ at 5 years. OS for NRSTS $=69,3(95 \% \mathrm{CI}$ $55,8-82,8)$.

Epidemiology studies of childhood cancer are hampered by the lack of population-based cancer registries in Russia. This study is the first published report presenting detailed analysis of epidemiological characteristics of STS in children in RF. Childhood population-based cancer registry data were used for analysis that precludes selection bias, typical for hospitalbased studies.

Children $<15$ years of age were included. It should be noted that in the literature different age intervals for the definition of "pediatric" population are used. Ferrari et al. [11] use this term for cases aged $<20$ years. On the contrary, Automated Childhood Cancer Information System (ACCIS) in Europe separately analyzes cases in children 0-14 years old and adolescents 15-19 years old [12].

The incidence of STS in children varies considerably depending on geographic region and ethnic group [2]. 
TABLE 2: Absolute number of cases, crude, ASR, and age-specific incidence rate of soft tissue sarcomas by ICCC-3 in children (0-14 years) diagnosed in Moscow Region in 2000-2009.

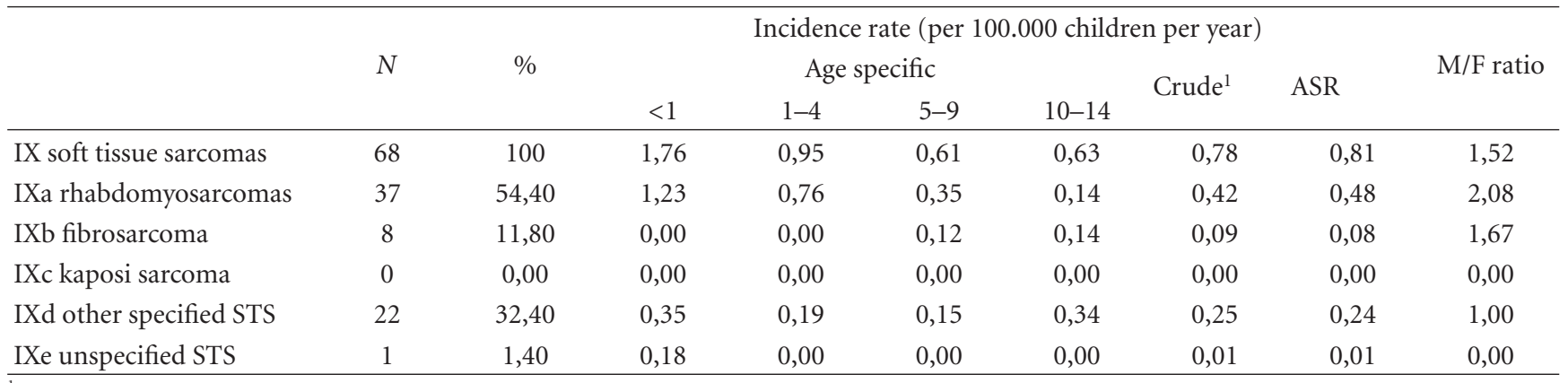

${ }^{1}$ The sum does not correspond to 0,78 due to rounding in calculations.

TABLE 3: Frequency and percentage of STS by site and histology.

\begin{tabular}{|c|c|c|c|c|c|c|c|}
\hline \multirow{2}{*}{ Site of tumor } & \multicolumn{2}{|c|}{ All STS } & \multicolumn{2}{|c|}{ RMS } & \multicolumn{2}{|c|}{ NRSTS } & \multirow{2}{*}{$\begin{array}{l}\text { RMS versus NRSTS } \\
\text { (Fisher's exact test) }\end{array}$} \\
\hline & $N$ & $\%$ & $N$ & $\%$ & $N$ & $\%$ & \\
\hline Head and neck & 17 & 25,0 & 11 & 29,8 & 6 & 19,3 & n.s. ${ }^{2}$ \\
\hline Orbit & 5 & 7,3 & 5 & 13,5 & 0 & 0 & n.s. \\
\hline Pelvis & 8 & 11,8 & 6 & 16,2 & 2 & 6,5 & n.s. \\
\hline Genitourinary system & 6 & 8,8 & 6 & 16,2 & 0 & 0 & $P=0,0279$ \\
\hline Limbs & 17 & 25,0 & 3 & 8,1 & 14 & 45,2 & $P=0,0005$ \\
\hline Thorax & 4 & 5,9 & 1 & 2,7 & 3 & 9,7 & n.s. \\
\hline Others & 10 & 14,7 & 4 & 10,8 & 6 & 19,3 & n.s. \\
\hline $\mathrm{NOS}^{1}$ & 1 & 1,5 & 1 & 2,7 & 0 & 0 & n.s. \\
\hline Total & 68 & 100,0 & 37 & 100,0 & 31 & 100,0 & \\
\hline
\end{tabular}

${ }^{1}$ NOS: non otherwise specified; ${ }^{2}$ n.s.: not significant.

In general, higher incidence rates were observed in Europe and North America, lower ones in Asian countries. Data from the International Agency for Research on Cancer (IARC) showed that incidence rate of STS in Caucasians children varied from 0,5 to 0,9 per 100.000 per year [2]. According to ACCIS average incidence rate of STS in children $<15$ years in Europe is 0.91 per 100.000 per year, and it varies between 5 geographic regions [12]. The highest incidence rate of STS is observed in Northern countries $(1,1$ per 100.000 children per year), the lowest in Eastern Europe (0.84 per 100.000 children per year). The cause of these geographical differences is not clear; however, the impact of variations in diagnostic, classification, and registration criteria could not be ruled out [12]. The incidence rate of STS obtained in our study (0,81 per 100.000 children per year) was comparable to the data of the Eastern European countries included in ACCIS project. It reflects the completeness of childhood STS registration by childhood population-based cancer registry in MR.

Certain congenital anomalies and genetic conditions (e.g., Li-Fraumeni syndrome, neurofibromatosis type I) are known as the strongest risk factors, although they explain only a small proportion of cases $[3,13]$. Thus, Narod et al. reported cancer predisposition syndromes in $20(2,0 \%)$ out of 1003 children with STS in Great Britain [14]. Radiation therapy is the best-known environmental risk factor for
STS [15]. HIV infection is responsible for cases of Kaposi's sarcoma in Africa [2]. Kaposi's sarcoma makes up about twothirds of STS and $20 \%$ of all childhood cancers in some African countries [16] but only 1\% of STS in the US [3].

In our study genetic predisposition syndromes were revealed in $4,4 \%$ cases and were presented by neurofibromatosis type 1 . In 1 patient with hereditary retinoblastoma RMS developed as a second malignant neoplasm in the radiation field. No cases of Kaposi's sarcoma in our study were detected, which is consistent with the data of the European and North American cancer registries, indicating extreme rarity of the disease $[3,12]$.

The incidence rate of STS in children varies by age. The highest incidence seen in infants (1,3-2,1 per 100.000) gradually decreases to $0,6-0,8$ per 100.000 per year in $5-9$ year age group, with further increase to $0,72-1,1$ per 100.000 per year in children older than 10 years $[3,12]$. The results of our study also corroborate with the previous findings: the highest incidence observed in infants (1,76 per 100.000 per year), the lowest in 5-9-year age group (0,61 per 100.000 per year). The biological basis of age-dependent differences in incidence rate is not clear. It is speculated that higher STS incidence in young children could be a result of genetic predisposition. Diller et al. in a study of 33 cases of sporadic RMS showed that 3 of 13 children younger than 3 years of age at diagnosis (compared with none of the 20 children older 
than 3 years of age) had germline mutations in their p53 gene [17].

The distribution of specific subgroups of STS according to ICCC-3 showed prevalence of RMS (IXa) and the subgroup of "other specified STS" (IXd). The most extensive studies on STS epidemiology in children $[2,3,12]$ were based on International Classification of Childhood Cancer, $2 \mathrm{~d}$ ed. (ICCC-2) [18]. Several histological types (e.g., pleomorphic malignant fibrous histiocytoma) assigned to a subgroup of "fibrosarcoma" (IXb) in ICCC-2 were included into subgroup of "other specified STS" (IXd) [6]. It complicates a comparative analysis of different STS subgroups because in our study tumors were stratified according to ICCC-3. Nevertheless, recent report of German Childhood Cancer Registry based on ICCC-3 [7] confirmed our findings. RMS and "other specified STS" were the two most common groups of STS in children comprising 58,3\% and 27,3\%, respectively [7]. RMS represents the single most common histological type of STS in our childhood population. This observation is consistent with the population-based data from other countries $[3,7]$. Age-related changes in ratio of RMS and NRSTS identified in children in MR have been previously described by others $[3,7,12]$. Data from German Childhood Cancer Registry showed decreased percentage of RMS in older age groups: 71, 64 and $34 \%$ in children 0 $4,5-9$, and 10-14 years of age, respectively [7]. Correlation of histological tumor type with the topography of primary tumor is well known [1]. As expected the most frequent localization of RMS was the head and neck region, whereas NRSTS predominantly affected limbs.

Analysis of survival of children with cancer at the population level is an extremely important index reflecting the quality of specialized medical care. Data from ACCIS project showed significant differences in survival of children with STS in Europe [12]. Thus, for the pooled European data 5 -year survival of patients with STS diagnosed in 19881997 years was 65\%, ranging from 74\% in Northern and Southern Europe to 50\% in Eastern Europe [12]. The average survival rate of children with RMS in Europe was 63\%, with the lowest survival observed in Eastern Europe-40\%. In the recently published report of Eurocare project 5-year survival of children with RMS in Eastern Europe was 64,9\% [19]. Data from the USA Surveillance Epidemiology and End Results program (SEER) showed 5-year survival rate of patients registered in 2001-2007, 72,9\% for the entire group of STS, and $68.1 \%$ for RMS [20]. 5-year survival of children with STS, obtained in this study, was $64,1 \%$ for the whole group of STS and 59,2\% for RMS and was lower in Western countries but compatible to the data from East Europe. It should be noted that STS represents significant challenges for treatment and requires a multidisciplinary approach to therapy. Protocol-based therapy of cancer in children, including STS, was introduced in MR only in 2000, that definitely has affected treatment results obtained in the present study. Age is an important prognostic factor in STS, especially in RMS $[1,11,12,21]$. The worst results of therapy have been reported in infants and children over 10 years of age at the time of diagnosis. According to ACCIS data the better survival was observed in children aged 5-9-year $(70 \%)$, whereas in other age groups survival ranged from $57 \%$ in infants up to $64 \%$ in children aged $1-$ 4 years [12]. In our study there was a trend towards better survival of children in 5-9 years age group (5-year OS $80 \%$ ), but difference was not statistically significant due to small number of patients.

In conclusion, the present study provides the first data on incidence and survival of STS in children in RF. The epidemiological pattern of the disease is compatible with the data from Europe and North America. Small number of patients is one of the main limitations of this study. On the contrary, strength of this analysis includes population-based data and accuracy of the data confirmed by microscopic verification in all cases, lack of death certificate cases, and low number of patients assigned to the subgroup "unspecified STS". The obtained data can form a basis for development of population cancer control programs for children with cancer in other regions of RF.

\section{References}

[1] W. H. Meyer and S. L. Spunt, "Soft tissue sarcomas of childhood," Cancer Treatment Reviews, vol. 30, no. 3, pp. 269-280, 2004.

[2] C. A. Stiller and D. M. Parkin, "International variations in the incidence of childhood soft-tissue sarcomas," Paediatric and Perinatal Epidemiology, vol. 8, no. 1, pp. 107-119, 1994.

[3] J. G. Gurney, J. L. Young Jr., S. D. Roffers et al., "Soft tissue sarcomas," in Cancer Incidence and Survival among Children and Adolescents: United States SEER Program 1975-1995, L. A. G. Ries, M. A. Smith, and J. G. Gurney, Eds., pp. 111123, National Cancer Institute, SEER Program, Bethesda, Md, USA, 1999.

[4] P. Kaatsch and C. Spix, German Childhood Cancer RegistryAnnual Report 2006/07 (1980-2006), Institute of Medical Biostatistics, Epidemiolgy and Informatics, University of Mainz, Mainz, Gemany, 2008.

[5] A. Fritz, C. Percy, A. Jack et al., International Classification of Diseases for Oncology, World Health Organization, Geneva, Switzerland, 3rd edition, 2000.

[6] E. Steliarova-Foucher, C. Stiller, B. Lacour, and P. Kaatsch, "International classification of childhood cancer, third edition," Cancer, vol. 103, no. 7, pp. 1457-1467, 2005.

[7] T. Weihkopf, M. Blettner, T. Dantonello et al., "Incidence and time trends of soft tissue sarcomas in German children 1985-2004-a report from the population-based German Childhood Cancer Registry," European Journal of Cancer, vol. 44, no. 3, pp. 432-440, 2008.

[8] T. M. Dantonello, C. Int-Veen, D. Harms et al., "Cooperative trial CWS-91 for localized soft tissue sarcoma in children, adolescents, and young adults," Journal of Clinical Oncology, vol. 27, no. 9, pp. 1446-1455, 2009.

[9] P. Boyle and D. M. Parkin, "Statistical methods for registries," in Cancer Registration. Principles and Methods, O. M. Jensen, D. M. Parkin, R. MacLennan, C. S. Muir, and R. G. Skeet, Eds., pp. 126-158, IARC Scientific, Lyon, France, 1991.

[10] E. L. Kaplan and P. Meier, "Nonparametric estimation from incomplete observations," Journal of the American Statistical Association, vol. 53, pp. 457-481, 1958.

[11] A. Ferrari, I. Sultan, T. T. Huang et al., "Soft tissue sarcoma across the age spectrum: a population-based study from the 
surveillance epidemiology and end results database," Pediatric Blood and Cancer, vol. 57, no. 6, pp. 943-949, 2011.

[12] G. Pastore, R. Peris-Bonet, M. Carli, C. Martínez-García, J. S. de Toledo, and E. Steliarova-Foucher, "Childhood soft tissue sarcomas incidence and survival in European children (1978-1997): report from the Automated Childhood Cancer Information System project," European Journal of Cancer, vol. 42, no. 13, pp. 2136-2149, 2006.

[13] P. Yang, S. Grufferman, M. J. Khoury et al., "Association of childhood rhabdomyosarcoma with neurofibromatosis type I and birth defects," Genetic Epidemiology, vol. 12, no. 5, pp. 467-474, 1995.

[14] S. A. Narod, C. Stiller, and G. M. Lenoir, "An estimate of the heritable fraction of childhood cancer," British Journal of Cancer, vol. 63, no. 6, pp. 993-999, 1991.

[15] G. Bisogno, G. Sotti, Y. Nowicki et al., "Soft tissue sarcoma as a second malignant neoplasm in the pediatric age group," Cancer, vol. 100, no. 8, pp. 1758-1765, 2004.

[16] C. Chintu, U. H. Athale, and P. S. Patil, "Childhood cancers in Zambia before and after the HIV epidemic," Archives of Disease in Childhood, vol. 73, no. 2, pp. 100-105, 1995.

[17] L. Diller, E. Sexsmith, A. Gottlieb, F. P. Li, and D. Malkin, "Germline p53 mutations are frequently detected in young children with rhabdomyosarcoma," The Journal of Clinical Investigation, vol. 95, no. 4, pp. 1606-1611, 1995.

[18] E. Kramárová and C. A. Stiller, "The international classification of childhood cancer," International Journal of Cancer, vol. 68, no. 6, pp. 759-765, 1996.

[19] G. Gatta,, G. Zigon, R. Capocaccia et al., "Survival of European children and young adults with cancer diagnosed 1995-2002," European Journal of Cancer, vol. 45, no. 6, pp. 992-1005, 2009.

[20] N. Howlader, A. M. Noone, M. Krapcho et al., Eds., SEER Cancer Statistics Review, 1975-2008, National Cancer Institute, Bethesda, MD, USA, 2011.

[21] C. A. Stiller, M. C. G. Stevens, C. Magnani, and I. Corazziari, "Survival of children with soft-tissue sarcoma in Europe since 1978: results from the EUROCARE study," European Journal of Cancer, vol. 37, no. 6, pp. 767-774, 2001. 


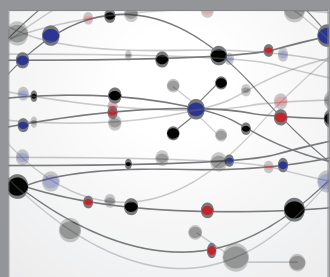

The Scientific World Journal
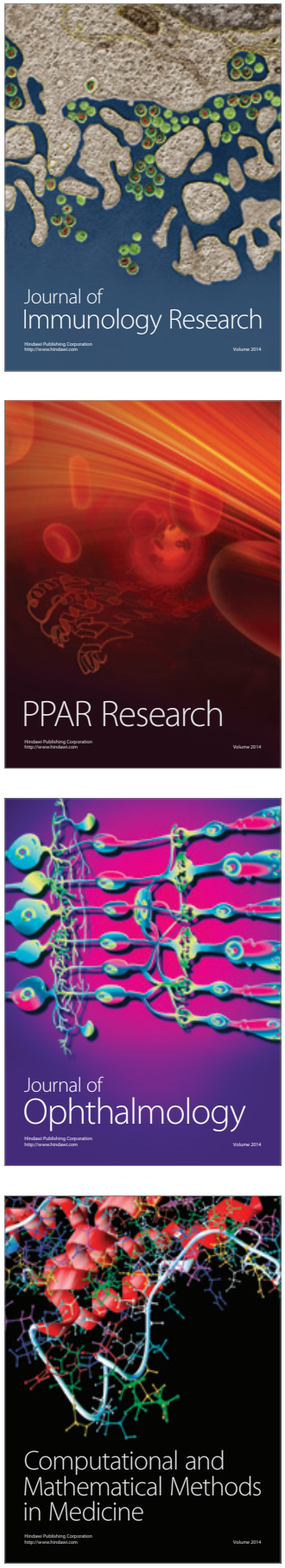

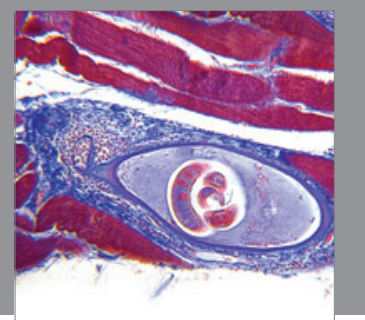

Gastroenterology

Research and Practice
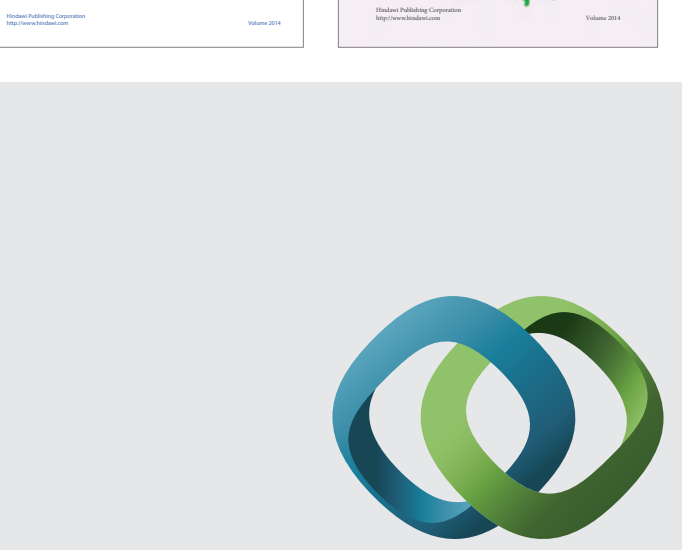

\section{Hindawi}

Submit your manuscripts at

http://www.hindawi.com
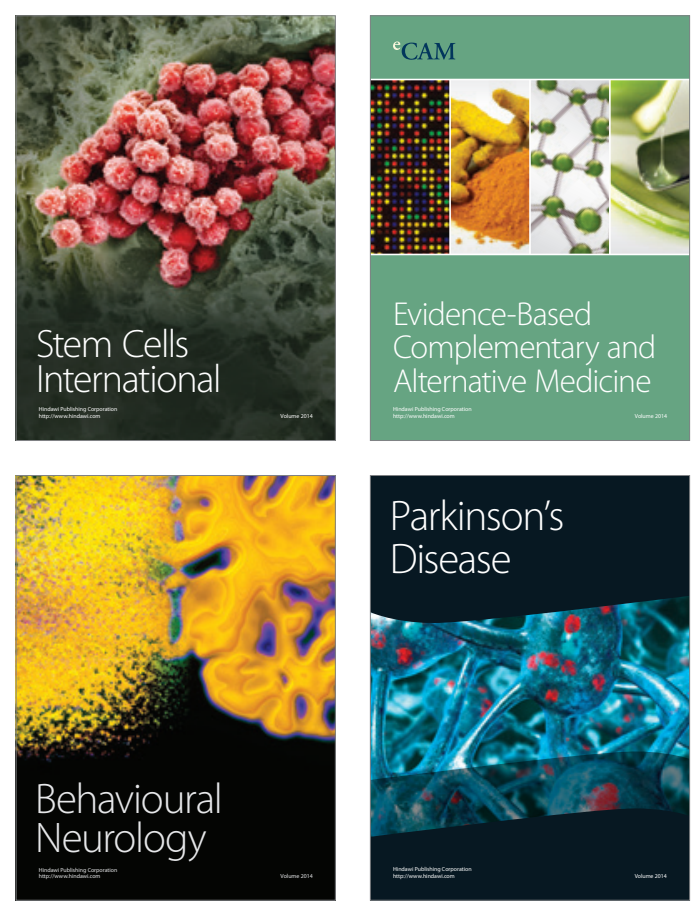

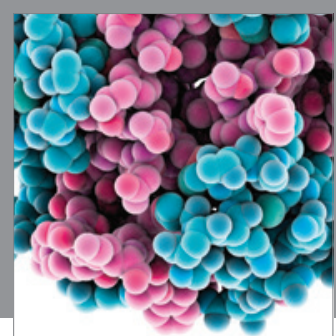

Journal of
Diabetes Research

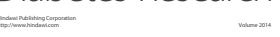

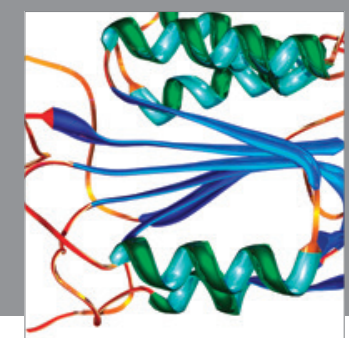

Disease Markers
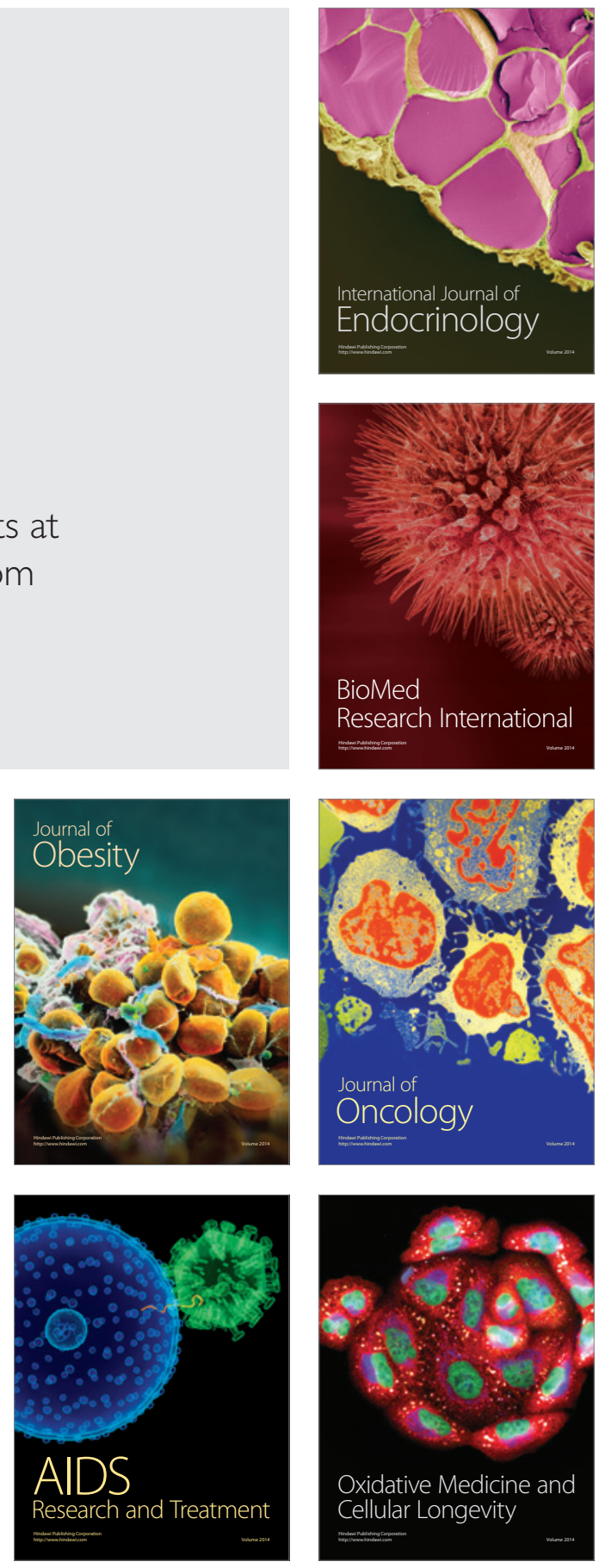